\title{
Association of genetic and immuno- characteristics with clinical outcomes in patients with RET-rearranged non-small cell lung cancer: a retrospective multicenter study
}

Chang $\mathrm{Lu}^{1,2+}$, Xiao-Rong Dong ${ }^{3 \dagger}$, Jun Zhao ${ }^{4 \dagger}$, Xu-Chao Zhang ${ }^{2}$, Hua-Jun Chen ${ }^{2}$, Qing Zhou ${ }^{2}$, Hai-Yan Tu' ${ }^{2}$, Xing-Hao Ai ${ }^{5}$, Xiao-Feng Chen ${ }^{6}$, Gai-Li An ${ }^{7}$, Jun Bai ${ }^{7}$, Jin-Lu Shan ${ }^{8}$, Yi-Na Wang ${ }^{9}$, Shuan-Ying Yang ${ }^{10}$, Xiang Liu ${ }^{11}$, Wu Zhuang ${ }^{12}$, Hui-Ta Wu ${ }^{13}$, Bo Zhu ${ }^{14}$, Xue-Feng Xia ${ }^{15}$, Rong-Rong Chen ${ }^{15}$, De-Jian Gu${ }^{15}$, Hua-Min Xu' ${ }^{15}$, Yi-Long $\mathrm{Wu}^{1,2^{*}}$ and Jin-Ji Yang ${ }^{1,2^{*}}$

\begin{abstract}
Background: Rearranged during transfection (RET) has been proven to be a tumorigenic target in non-small cell lung cancers (NSCLCS). In RET-rearranged NSCLCs, molecular features and their impact on prognosis were not well illustrated, and the activity of mainstay therapeutics has not currently been well compared.

Methods: Patients diagnosed with NSCLCs with RET rearrangements were analyzed for concomitant mutations, tumor mutation burden (TMB), PD-L1 expression, T cell receptor repertoire and clinical outcomes with chemotherapy, immune checkpoint inhibitors (ICIs), and multikinase inhibitors (MKIs).

Results: Among 129 patients with RET-rearranged NSCLC who were analyzed, 41.1\% (53/129) had co-occurring genetic alterations by next-generation sequencing, and concomitant TP53 mutation appeared most frequently (20/ 53, 37.7\%). Patients with concurrent TP53 mutation $(n=15)$ had shorter overall survival than those without $(n=30$; median, 18.4 months [95\% Cl, 8.6-39.1] vs 24.8 months [95\% Cl, 11.7-52.8]; $P<0.05$ ). Patients with lower peripheral blood TCR diversity $(n=5)$ had superior overall survival compared with those with higher diversity $(n=6$; median, 18.4 months [95\% Cl, 16.9-19.9] vs 4.8 months [95\% Cl, 4.5-5.3]; $P=0.035$ ). An association with overall survival was not observed for PD-L1 expression nor for tumor mutation burden level. Median progression-free survival was not significantly different across chemotherapy, ICls, and MKIs (median, 3.5 vs 2.5 vs 3.8 months). For patients treated with $\mathrm{ICls}$, the disease control rate was $60 \%(6 / 10)$ and the objective response rate was $20 \%(2 / 10)$.
\end{abstract}

(Continued on next page)

\footnotetext{
* Correspondence: syylwu@live.cn; yangjinji@gdph.org.cn

${ }^{+}$Chang Lu, Xiao-Rong Dong and Jun Zhao contributed equally to this work.

${ }^{1}$ The Second School of Clinical Medicine, Southern Medical University, Guangzhou, China

Full list of author information is available at the end of the article
}

(c) The Author(s). 2020 Open Access This article is licensed under a Creative Commons Attribution 4.0 International License, which permits use, sharing, adaptation, distribution and reproduction in any medium or format, as long as you give appropriate credit to the original author(s) and the source, provide a link to the Creative Commons licence, and indicate if changes were made. The images or other third party material in this article are included in the article's Creative Commons licence, unless indicated otherwise in a credit line to the material. If material is not included in the article's Creative Commons licence and your intended use is not permitted by statutory regulation or exceeds the permitted use, you will need to obtain permission directly from the copyright holder. To view a copy of this licence, visit http://creativecommons.org/licenses/by/4.0/ The Creative Commons Public Domain Dedication waiver (http://creativecommons.org/publicdomain/zero/1.0/) applies to the data made available in this article, unless otherwise stated in a credit line to the data. 
(Continued from previous page)

Conclusions: RET-rearranged lung cancers can be heterogeneous in terms of concomitant genetic alterations. Patients with concurrent TP53 mutation or high peripheral blood TCR repertoire diversity have relatively inferior overall survival in this series. Outcomes with traditional systemic therapies in general are suboptimal.

Keywords: Advanced NSCLC, RET rearrangement, Next-generationsequencing, TP53, Immune checkpoint inhibitor

\section{Background}

The dawn of the targeted therapy era saw the discovery of receptor tyrosine kinase RET fusion in $1-2 \%$ of nonsmall cell lung cancers (NSCLC) $[1,2]$ and proved it to be tumorigenic and targetable. Regarding the tumorigenicity, although several studies reported the prevalence of concomitant genetic alterations based on a limited sample size [3-6], the effects of these concomitant alterations on clinical outcomes were scant.

Regarding the druggability, since more specific and potent TKIs targeting RET such as BLU-667 and LOXO-29 $2[7-9]$ are currently not available for all of the patients, the common systemic treatment regimen now includes multikinase inhibitors (MKIs), chemotherapy, and immune checkpoint inhibitors (ICIs). The success of traditional MKIs is relatively limited [10-14]. The median progression-free survival (PFS) of the pemetrexed/platinum regimen was 19 months, 7.5 months, and 6.4 months in a single center [15], a Chinese cohort [5], and an international cohort [10], respectively. Although ICIs have been widely accepted, the outcomes of these treatment strategies in $R E T$-altered patients have not currently been well compared, and the immuno-characteristics in those patients have also not been well characterized in previous studies $[16,17]$.

Here, we describe genetic and immune profiling in a multicenter cohort of patients with RET-rearranged NSCLC, analyze their associations with clinical outcomes, and document treatment outcomes in routine clinical care.

\section{Methods}

\section{Study design and patients}

The study flow chart is shown in Supplementary figure 1 . This retrospective observational study was performed at 13 centers in China and included patients who had a pathologic diagnosis of NSCLC of any age with RET rearrangement determined by at least one of the validated tests including fluorescence in situ hybridization, reverse transcriptase polymerase chain reaction, and nextgeneration sequencing (NGS). Patients with acquired RET rearrangement after progression on EGFR TKIs were excluded due to the concern of the potential prognostic implications of frontline EGFR-TKI administration (RET cohort). This multicenter network of thoracic oncologists also identified EGFR/ALK/ROS1/pan-negative patients determined by targeted DNA sequencing performed in one institute (EGFR/ALK/ROS1/pan-negative cohort). There is no overlap between these cohorts. Only patients with locally advanced or metastatic NSCLC were analyzed for clinical outcomes. Written consent and institutional approval were obtained.

\section{Genotyping and immunotyping}

Commercial targeted DNA sequencing (Geneplus or Burning Rock or Geneseeq) was used to calculate genetic alterations and tumor mutation burden (TMB). More than $90 \%$ of the samples were sequenced in one institute. Our genetic profiling platform is designed and validated to categorize point mutations, insertions, deletions, copy number variations, and rearrangements. PDL1 levels by immunohistochemistry were assessed by one local laboratory as previously described [18].

\section{DNA extraction and processing}

Genomic DNA of tissue samples was extracted by using the QIAamp DNA FFPE Tissue Kit or the DNeasy Blood \& Tissue Kit (Qiagen, Hilden, Germany). For cell-free DNA (cfDNA) extraction, plasma was separated by centrifugation at $1600 \times g$ for $10 \mathrm{~min}$, then transferred to a new microcentrifuge tube and centrifuged at $16,000 \times g$ for another $10 \mathrm{~min}$ to remove any remaining cell debris. cfDNA was isolated from the plasma using the QIAamp Circulating Nucleic Acid Kit (Qiagen, Hilden, Germany). Peripheral blood lymphocytes (PBLs) were used to extract germline genomic DNA from each patient with the DNeasy Blood \& Tissue Kit (Qiagen, Hilden, Germany). A Qubit fluorometer and the Qubit dsDNA HS (High Sensitivity) Assay Kit (Invitrogen, Carlsbad, CA USA) were used for DNA concentration measurement. And the size distribution of cfDNA was assessed with an Agilent 2100 BioAnalyzer and the DNA HS kit (Agilent Technologies, Santa Clara, CA, USA).

\section{Library construction and target capture sequencing}

We used protocols recommended in the Illumina TruSeq DNA Library Preparation Kit (Illumina, San Diego, CA) for the construction of the Indexed Illumina NGS libraries. About 20-80 ng cfDNA per sample was used. For genomic DNA extracted from either tissue or PBLs, about $1 \mu \mathrm{g}$ DNA was sheared with a Covaris S2 ultrasonicator (Covaris, Woburn, MA, USA) to generate fragments with 
a peak of $250 \mathrm{bps}$ for library construction. Then end repair, tailing, and ligation to the Illumina-indexed adapters were done according to the standard library construction protocol. The constructed libraries were hybridized to custom-designed biotinylated oligonucleotide probes (Roche NimbleGen, Madison, WI, USA) for target enrichment. The probes cover 1021 cancer-related genes (Supplementary table 1). The captured DNA fragments were amplified and pooled to generate multiplex libraries. Then sequencing was done using Illumina $2 \times 75$ bp paired-end reads with the HiSeq 3000 Sequencing System (Illumina, San Diego, CA).

\section{Sequencing data analysis}

After removing terminal adaptor sequences and lowquality reads, the clean reads were mapped and aligned to the reference human genome (hg19) with BWA (version 0.7.12-r1039) [19]. MuTect2 (3.4-46-gbc02625) [20] was used to call single nucleotide variants (SNVs) while GATK was employed to call small insertions and deletions (Indels). Copy number variations (CNVs) were detected using Contra (2.0.8) [21]. And structure variations (SVs) were detected with BreakDancer. All final candidate variants were verified with the integrative genomics viewer browser. TMB was defined as the number of somatic nonsynonymous mutations per megabase including SNVs, insertions, and deletions of the panel region [22].

\section{$T$ cell receptor sequencing and data analysis}

The $\mathrm{T}$ cell receptor (TCR) repertoire has recently emerged as a novel biomarker [23, 24]. Previous pilot studies showed that tumor-infiltrated TCR clonality [25] and peripheral blood $\mathrm{T}$ cell receptor repertoire diversity [26-28] could have a potential role as predictors of the response to ICI therapy. We conducted multiplex PCR amplification on complementarity-determining region 3 (CDR3), a hypervariable region of the TCR $\beta$ chain that is unique to each TC R[29] as previously described [27]. The diversity metric accounts for both "richness" and "evenness" components, while richness is a measurement of the number of different specificities in the sample (e.g., the number of $\mathrm{T}$ cell clones with unique TCRs), evenness measures the relative abundance of these different specificities. Diversity can be measured in many ways; one of them uses Shannon's entrop y[30], in which higher diversity values indicate a more diverse distribution of the receptor sequences. The evenness or relative abundance metric can be calculated in different manners, such as Pielou's evenness, originally developed for measurements derived from ecology [31]. Clonality, a metric of $\mathrm{T}$ cell expansion and reactivity, ranges from 0 to 1 and describes the shape of the $\mathrm{T}$ cell frequency distribution: clonality values approaching 0 indicate a very even distribution of clone frequencies, whereas values approaching 1 indicate an increasingly asymmetric distribution in which a few clones are present at high frequencies [32]. The diversity and clonality of the tissue and blood TCR repertoire are represented T-Shannon, T-clonality, T-evenness, B-Shannon, B-clonality, and Bevenness, respectively, in this manuscript.

\section{Outcomes}

Data on clinical treatments and outcomes were collected since advanced diagnosis. Overall survival was defined as the time between the date of advanced diagnosis to the date of death from any cause or last follow-up. PFS was measured from the start of treatment to disease progression, death from any cause or last follow-up. Disease control rate (DCR) was defined as the proportion of achieving disease control (stable disease or radiologically confirmed complete/partial response). The objective response rate (ORR) was defined as the proportion achieving an objective response (radiologically confirmed complete/partial response). The investigators and the treating physicians ascertained tumor response according to the Response Evaluation Criteria in Solid Tumors (RECIST) version 1.1 and iRECIST (a modified RECIST 1.1 for immune-based therapeutics). The median follow-up time was 12.7 months and the last follow-up date was June 28, 2019.

\section{Statistics}

The data were analyzed by GraphPad Prism 8.0 and SPSS Statistics 19. A $P$ value $<0.05$ was considered statistically significant. The median TMB of RET-rearranged NSCLCs was compared with that of $A L K$-rearranged, ROS1-rearranged, EGFR-mutant NSCLCs from the screened population using the Mann-Whitney test. Patients, irrespective of ICI treatment in their disease course, were stratified by cutoff values of the Shannon, evenness, and clonality indexes. The cut-off values were determined by ROC analysis. Kaplan-Meier analysis was used to estimate PFS and overall survival, presented as median values. The log-rank test was used to compare the curves. Hazard ratios were calculated using the log-rank method. Spearman's rank test was used to estimate correlations between the TCR repertoire indexes and overall survival. Baseline variables that were considered clinically relevant or that showed a univariate relationship with outcomes were entered into the multivariate Cox proportional hazards model (forward stepwise).

\section{Results}

\section{Clinicopathologic and molecular characteristics}

We retrospectively included 129 patients with $R E T$-rearranged lung cancer from 13 centers. The majority of patients presented stage III-IV disease at initial diagnosis $(n=110,85.3 \%)$. Patients were preponderantly never smokers $(n=58,45.0 \%)$ and had adenocarcinoma histology $(n=112,86.8 \%)$ with a median age of 57 years 
(range 24-82 years) and a sex makeup of 51.9\% female and $48.1 \%$ male. The detailed clinical characteristics are provided in Table 1 , and the frequencies of missing data are also shown.

Of 129 patients with baseline genetic profiles, all had RETfusion by NGS or FISH, including 99 (76.0\%) with KIF5B$R E T, 24$ (18.6\%) with CCDC6-RET, and 2 (1.6\%) with NCOA4-RET. No patient harbored concurrent driver oncogenic alterations. The most common concomitant mutations identified in RET-positive NSCLC were TP53 (38\%) and SETD2 (9\%, Fig. 1a, b). Other co-alterations that have a potential impact on tumor biology include KEAP1 (4\%) and combined $C D K N 2 A / C D K N 2 B$ (4\%). TMB levels calculated based on a large panel of 1021 genes ranged from 1.4 to 25.9 muts $/ \mathrm{Mb}$ (median $=5.8$ ) in RET-rearranged NSCLCs. No significant difference in TMB level was found among alterations in RET and other fusion genes, such as $A L K$ (range 1.4 to 27.4 muts $/ \mathrm{Mb}$, median $=6.5)$ and ROS1 (ranged 1.4 to 21.6 muts $/ \mathrm{Mb}$, median $=4.3$ ). TMB level appeared higher in EGFR-mutant NSCLCs (range 1.4 to 77.8 muts/ $\mathrm{Mb}$, median $=10.1$ ) and pan-negative NSCLCs (range 1.4175.7, media $=13.7, \mathrm{p}<0.0001$ for both) (Fig. 1c). High ( $\geq$ $50 \%)$, intermediate (1-49\%), and negative (<1\%) PD-L1 expression was observed in 5/20 (25\%), 9/20 (45\%), and 6/20 (30\%) cases, respectively (Fig. 1d).

\section{Association of genetic and immuno-characteristics with clinical outcomes}

Analyses of overall survival were restricted to patients with advanced disease and those who had survival data $(n=45)$. In the entire cohort, the median overall survival from the initial advanced diagnosis was 20.3 months (95\% CI, 8.4-49.2) (Fig. 2a). In cancers with known upstream fusion partners, $R E T$ fusions involving KIF5B $(n=35)$ were not associated with a benefit on overall survival compared to those involving partners other than KIF5B ( $n=10$; median overall survival, 18.4 months
[95\% CI, 7.8-43.5] vs 20.3 months [95\% CI, 8.6-48.0]; $P=$ 0.58) (Fig. 2b). We analyzed the overall survival of patients with ICI treatment in their disease course to assess the prognostic implications of TMB. The results were limited by the small number of patients $(n=6)$ (Supplementary figure 2). Patients with concurrent TP53 mutation $(n=15)$ had shorter survival than those without $(n=30$; median overall survival, 18.4 months [95\% CI, 8.6-39.1] vs 24.8 months [95\% CI, 11.7-52.8]; $P<0.05$ ) (Fig. 2c). Notably, those harboring TP53 loss-of-function $(n=11$, including TP53 loss-offunction and likely loss-of-function) showed a more obvious survival disadvantage (median overall survival, 10.2 months vs 24.8 months; $P=0.0041$ ) (Fig. 2d, Supplementary table 2). Multivariable analysis of these patients revealed that concomitant TP53 mutation was an independent poor prognostic factor $(\mathrm{HR}=2.26[95 \% \mathrm{CI}$, $1.04-4.91] ; P=0.040$ ) (Table 2). An association with overall survival was not observed for PD-L1 expression.

We collected peripheral blood $(n=19)$ and tissue samples $(n=22)$ from patients with advanced NSCLC to assess the diversity and clonality of the TCR repertoire. Of these patients, 21 with known overall survival from advanced diagnosis were analyzed to assess the potential prognostic significance of baseline blood $(n=11)$ or tissue TCR $(n=10)$ repertoire diversity. Patients, irrespective of ICI treatment in their disease course, were stratified by the cut-off value of each of three indexes determined by ROC analysis. A significant negative correlation was observed between overall survival and B-Shannon index $(P=$ 0.014, Spearman $r=-0.727$; Fig. 3a). Patients with a lower B-Shannon index or higher B-clonality were associated with significantly longer overall survival than those with a higher B-Shannon index or lower B-clonality (Fig. 3d, f). However, this association with overall survival was not found in other indexes ( $\mathrm{T}$-Shannon, T-clonality, $\mathrm{T}$ evenness). In 24 samples with available data on

Table 1 Clinicopathologic features in patients with RET-positive NSCLC. Demographics of 129 patients with RET-rearranged lung cancers as well as patients with RET wild-type lung cancers

\begin{tabular}{|c|c|c|c|c|c|c|}
\hline & & $\operatorname{RET}(N=129)$ & $\operatorname{ALK}(N=160)$ & $\operatorname{ROS1}(\mathrm{N}=76)$ & EGFR $(N=214)$ & Pan negative $(\mathrm{N}=226)$ \\
\hline \multirow[t]{2}{*}{ Age (years) } & Median & 57 & 49 & 54 & 58 & 63 \\
\hline & Range & $24-82$ & $23-75$ & $25-83$ & $29-82$ & $25-83$ \\
\hline \multirow[t]{2}{*}{ Gender (\%) } & $\mathrm{F}$ & $67(51.9)$ & $87(54.4)$ & 41 (53.9) & $133(62.1)$ & $52(23.0)$ \\
\hline & $M$ & $62(48.1)$ & $73(45.6)$ & $35(46.1)$ & $81(37.9)$ & $174(77.0)$ \\
\hline \multirow[t]{6}{*}{ Histology subtype (\%) } & Adenocarcinoma & $112(86.8)$ & $158(98.8)$ & $73(96.1)$ & $212(99.1)$ & $167(73.9)$ \\
\hline & Squamous & $4(3.1)$ & $2(1.2)$ & $1(1.3)$ & $2(0.9)$ & $58(25.7)$ \\
\hline & Adenosquamous & $1(0.8)$ & - & - & - & - \\
\hline & SCLC & $1(0.8)$ & - & - & - & - \\
\hline & others & $1^{\#}(0.8)$ & - & $2^{5}(2.6)$ & - & $1^{*}(0.4)$ \\
\hline & NA & $10(7.8)$ & - & - & - & - \\
\hline \multirow[t]{3}{*}{ Clinical stage (\%) } & I II & $7(5.4)$ & $4(2.5)$ & $5(6.6)$ & $33(15.4)$ & $32(14.2)$ \\
\hline & III IV & $110(85.3)$ & $156(97.5)$ & $71(93.4)$ & $181(84.6)$ & $194(85.8)$ \\
\hline & NA & $12(9.3)$ & - & - & - & - \\
\hline \multirow[t]{3}{*}{ Smoking history (\%) } & Non-smokers & $58(45.0)$ & $58(36.3)$ & $47(61.8)$ & $100(46.7)$ & $58(25.7)$ \\
\hline & Smokers & $25(19.4)$ & $18(11.2)$ & $14(18.4)$ & 42 (19.6) & $86(38.0)$ \\
\hline & NA & $46(35.6)$ & $84(52.5)$ & 25 (19.7) & $72(33.6)$ & $82(36.3)$ \\
\hline
\end{tabular}



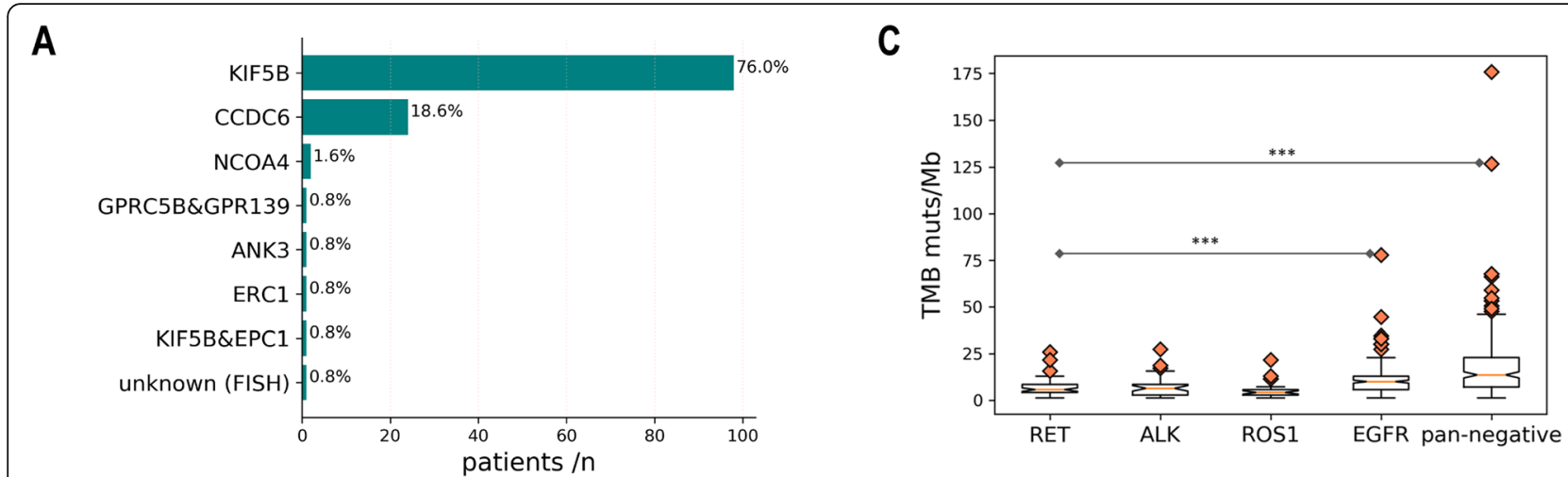

B
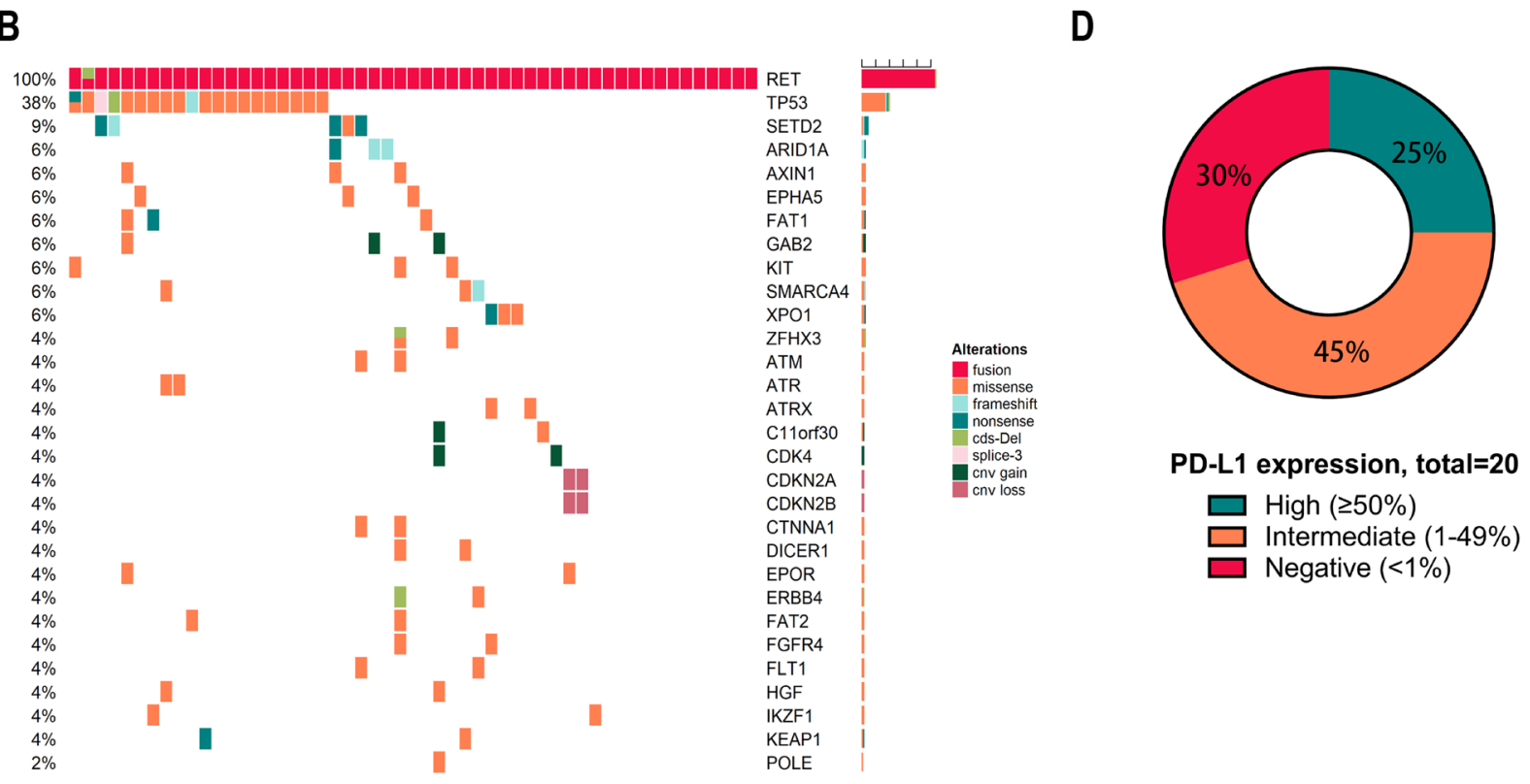

Fig. 1 Genotype and immunophenotype of RET-rearranged lung cancers. a RET upstream fusion partner. $\mathbf{b}$ Genomic alterations of patients with RET-positive NSCLC at the time they were diagnosed indicate that RET-rearrangement is mutually exclusive with common driver alterations. $\mathbf{c}$ Number of mutations of RET-rearranged lung cancers compared with ALKRROS1/EGFR-altered lung cancers and RET/ALK/ROS1/EGFR-negative lung cancers. d PD-L1 expression in RET-positive NSCLC. Abbreviations: TMB - tumor mutation burden

treatment, baseline blood $(n=11)$ or tissue $(n=13)$ TCR repertoire diversity did not indicate a significant association with PFS.

\section{Outcomes with MKIs, ICls, and chemotherapy}

After the advanced diagnosis, 45 patients received MKIs targeting RET ( $n=19)$, ICIs $(n=11)$, and chemotherapy $(n=29)$. Twelve of 45 patients received two or three of these treatments in different lines during disease courses. Notably, none of our patients received selective $R E T$ inhibitors, owing to the unavailability of these drugs in our country by the date of data cut-off. PFS across the three treatment groups is shown in Fig. 4a and demonstrated no significant difference among the groups (median PFS, MKIs, 3.8 months [95\% CI, 1.7-8.5], ICIs, 2.5 months [95\% CI, 1.1-5.8], chemotherapy, 3.5 months [95\% CI, 1.5-7.9]).
Eleven patients received ICIs in clinical trials or at their own expenses as first-line $(n=4)$, second-line $(n=4)$, and after second-line $(n=3)$ treatment. In 10 patients with evaluable response, DCR was $60 \%$, and ORR was $20 \%$. Three patients had durable PFS of 6.3, 10.4, and 11.5 months. Two of them were PD-L1 positive, while one patient lacked sample for IHC assessment. Changes in target lesions from baseline to best response, or the initial radiographic assessment, as well as treatment information of each patient (regimen, line, and response), are shown in Fig. 4b. The median tumor shrinkage/growth was $-2.4 \%$ (range $-44 \% \sim 23.3 \%$ ).

Two cases with KIF5B-RET fusion and high level of PD-L1 expression responding to $\mathrm{ICls}$

Case 1 was a 55-year-old female never smoker, with KIF5B-RET-rearranged lung adenocarcinoma and brain metastasis, was treated with second-line cabozantinib. 


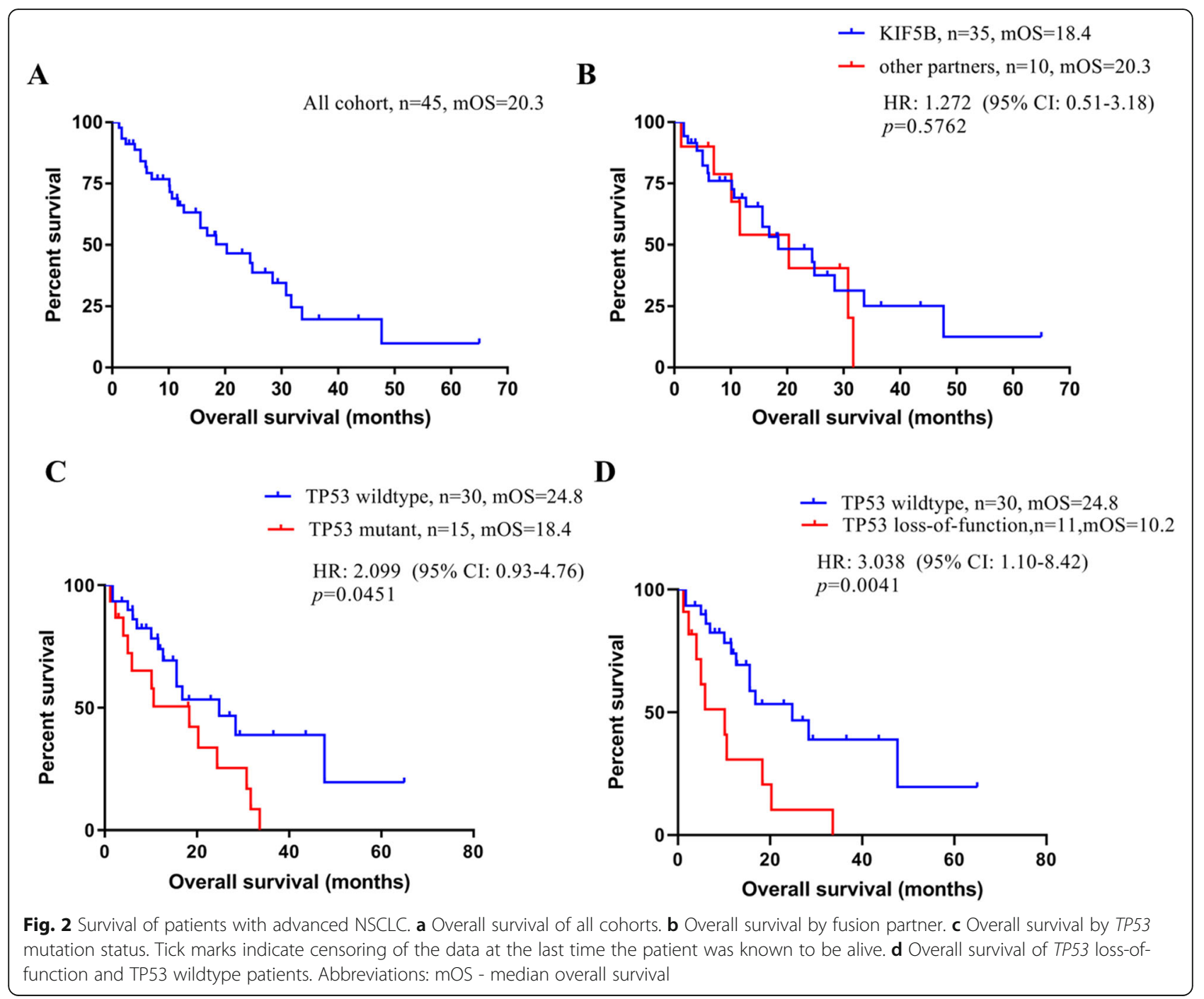

Four months later, intrathoracic progression was observed. Rebiopsy revealed lung adenocarcinoma with KIF5B-RET fusion and a high level of PD-L1 expression (TPS $=50 \%$; Fig. 5a, b). She was advised to start pembrolizumab thereafter. The patient achieved a confirmed partial response evaluated according to iRECIST and tumor reduction, both intrathoracic and intracranial PR, was noted (Fig. 5c).

Case 2 was a 74-year-old male with KIF5B-RET-rearranged stage IVA (cT1bN3M1b) lung adenocarcinoma.
Baseline tissue showed a high level of PD-L1 expression (TPS > 50\%). He was treated with first-line durvalumab and had confirmed partial response as the best response. Treatment is ongoing at the date of data cut-off.

\section{Discussion}

Despite the rarity of this driver gene, we report a relatively large sample of multicenter patients with RETaltered NSCLC with therapies commonly used in clinical practice. We demonstrate that this group of lung cancers

Table 2 Multivariate analysis of overall survival

\begin{tabular}{|c|c|c|c|c|c|c|c|}
\hline & \multicolumn{5}{|c|}{ Univariable analysis } & \multicolumn{2}{|c|}{ Multivariable analysis } \\
\hline & Tested & Reference & $\begin{array}{c}\text { Kaplan-Meier p } \\
\text { value }\end{array}$ & $\begin{array}{c}\text { Cox model HR } \\
(95 \% \mathrm{CI})\end{array}$ & p value & $\begin{array}{c}\text { Cox model HR } \\
(95 \% \mathrm{CI})\end{array}$ & p value \\
\hline Age & $\leqslant 60$ & $>60$ & 0.826 & $1.10(0.467-2.59)$ & 0.826 & & \\
\hline Sex & male & female & 0.053 & $2.11(0.97-4.57)$ & 0.058 & & \\
\hline Stage & III & IV & 0.868 & $1.19(0.16-8.93)$ & 0.869 & & \\
\hline TP53 & yes & no & 0.045 & $2.16(0.99-4.69)$ & 0.051 & $2.26(1.04-4.91)$ & 0.040 \\
\hline
\end{tabular}




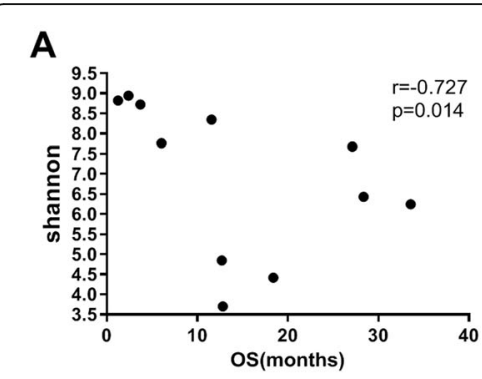

D

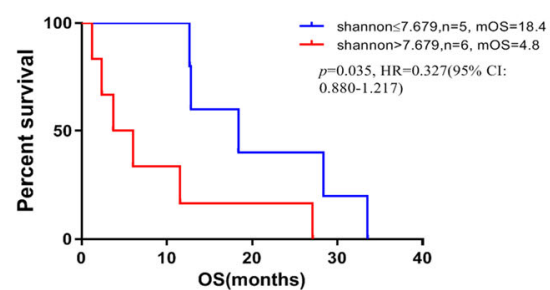

B

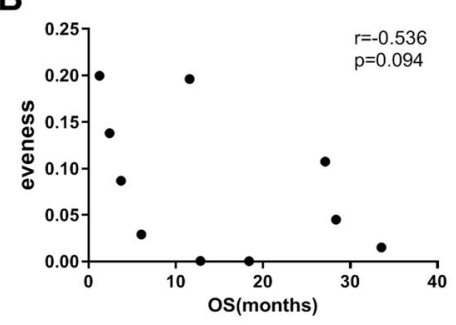

E

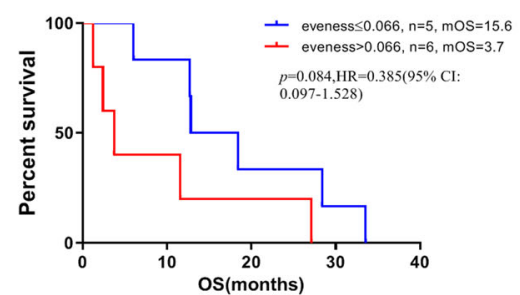

C

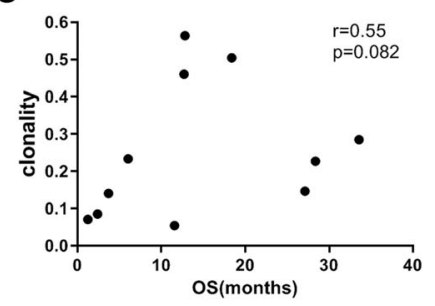

$\mathbf{F}$

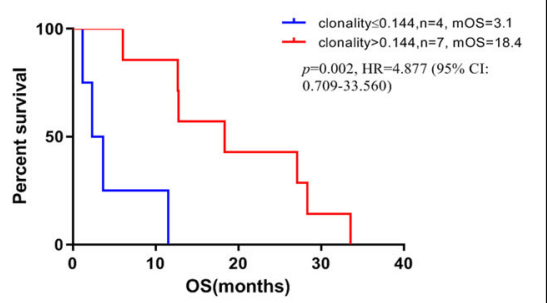

Fig. 3 Peripheral blood T cell receptor repertoire associated with overall survival. Correlation of B-Shannon (a, d), B-evenness (b, e), and Bclonality (c, f) of TCR CDR3s with the overall survival from advanced diagnoses. Spearman's rank test was used to estimate correlations between the TCR repertoire indexes and overall survival $(\mathbf{a}, \mathbf{b}, \mathbf{c})$. Kaplan-Meier analysis was used to estimate overall survival, presented as median values. The cut-off values of TCR repertoire indexes were determined by ROC analysis. The log-rank test was used to compare the curves (d, e, f). Abbreviations: mOS - median overall survival

is characterized by heterogeneous genotype and PD-L1 expression, as well as low TMB. Patients harboring concomitant TP53 were associated with inferior overall survival. We also found prognostic significance of TCR repertoire diversity in the peripheral blood. Although a subgroup of patients could benefit from ICIs, the optimal treatment option in routine clinical care remains to be defined.

In this study, we found an unfavorable clinical outcome in RET/TP53 co-mutated patients. Compared with other genes, TP53 co-mutations occur rather frequently with $R E T$ fusions. Recent works have suggested a negative impact of TP53 mutations on the outcomes of patients with EGFR-mutant [33, 34] and $A L K$-rearranged NSCLC [35, 36]. However, in RET-positive lung cancers, concomitant TP53 mutations have not been described as poor prognostic factors. The negative prognostic effect of TP53 mutations might be attributed to their tumorsuppressive function loss, genomic instability function gain, and abilities of cancer cell transcriptome and phenotype regulation [37]. Future research is warranted to improve the outcomes. In addition to TP53, other comutated genes, such as PIK3CA, etc., are also detected. All these findings call for an intensive study of the role of these additional genetic abnormalities in disease evolution and how they might influence the efficacy of treatments.

Whether RET fusion is mutually exclusive with other oncogenic drivers remains controversial. Recently, a study analyzing the fusion landscape in 33 cancer types highlighted the generally mutual exclusivity between fusions and mutations [38]. Nonetheless, Wang et al. [39] reported that one unique mutational signature in Chinese patients with NSCLC is associated with an increasing $E G F R$ mutation rate together with gene fusions, such as $R E T$ and $A L K$. In one retrospective analysis, concurrent EGFR mutations were found in 7 of $47 R E T$-rearranged adenocarcinomas [3]. In our study, patients with acquired RET-rearrangement after progression on EGFR TKIs were excluded due to the concern of the potential prognostic implications of frontline EGFR-TKI administration, and no co-existence of other driver-gene alteration appeared.

Previous studies have shown TCR repertoire diversity in the peripheral blood to be an indicator of prognosis, and high TCR repertoire diversity might indicate favorable outcomes [23, 27, 28]. Our study supplemented the prognostic value of TCR repertoire diversity in RETdriven lung cancers but disagree with the latter notion. Two explanations should be considered. First, although tumors with high TCR repertoire diversity are interpreted as biologically hot, two recent studies have indicated that intrinsic tumor reactivity of the intratumoral TCR repertoire of CD8 T cells can be limited and variable, and there are bystander CD8 $\mathrm{T}$ cells [40, 41]. Therefore, it seems that not all $\mathrm{T}$ cells are specific for tumor antigens in this study. Second, our data are limited in the dynamic analysis of the TCR repertoire during treatment and tumor evolution. As $\mathrm{T}$ cells can be easily isolated from patients' blood without losing much 


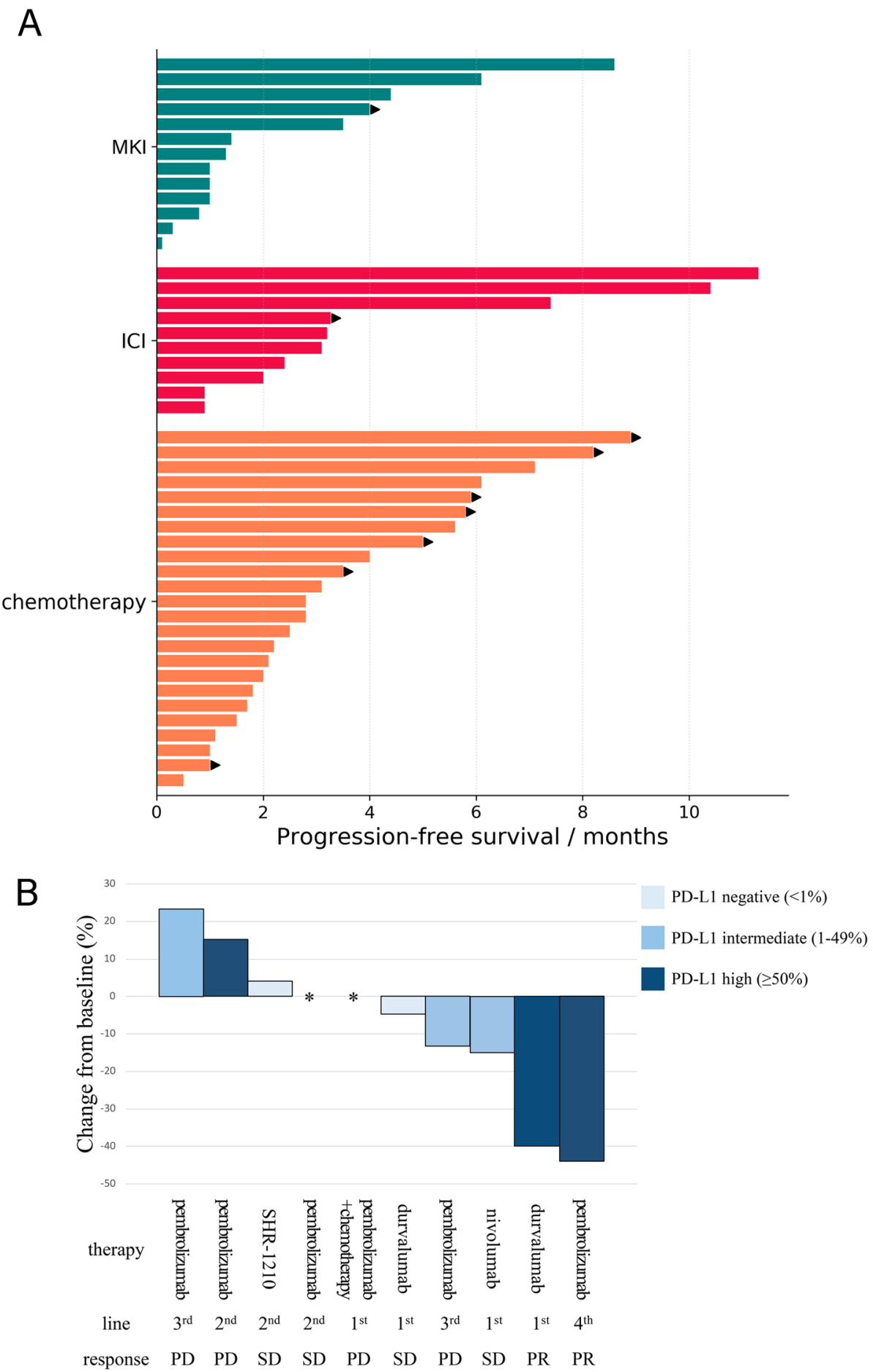

Fig. 4 Outcomes with diverse therapies. a Swimmer plot of PFS across chemotherapy, ICl, and TKI (all of which are MKIs) cohorts. The MKI regimen included cabozantinib $80 \mathrm{mg}$ qd, vandetanib $300 \mathrm{mg}$ qd, anlotinib $12 \mathrm{mg}$ qd, apatinib $500 \mathrm{mg}$ qd, and vandetanib 300 mg+everolimus $5 \mathrm{mg}$ qd. $\mathbf{b}$ Changes in target lesions from baseline to best response or the initial radiographic assessment, as well as treatment information (regimen, line, and response) and PD-L1 expression levels of 10 patients who received ICls. Abbreviations: PFS - progression-free survival, ICI immune checkpoint inhibitor, MKI - multi-kinase inhibitor, PD - progression disease, SD - stable disease, PR - partial response 

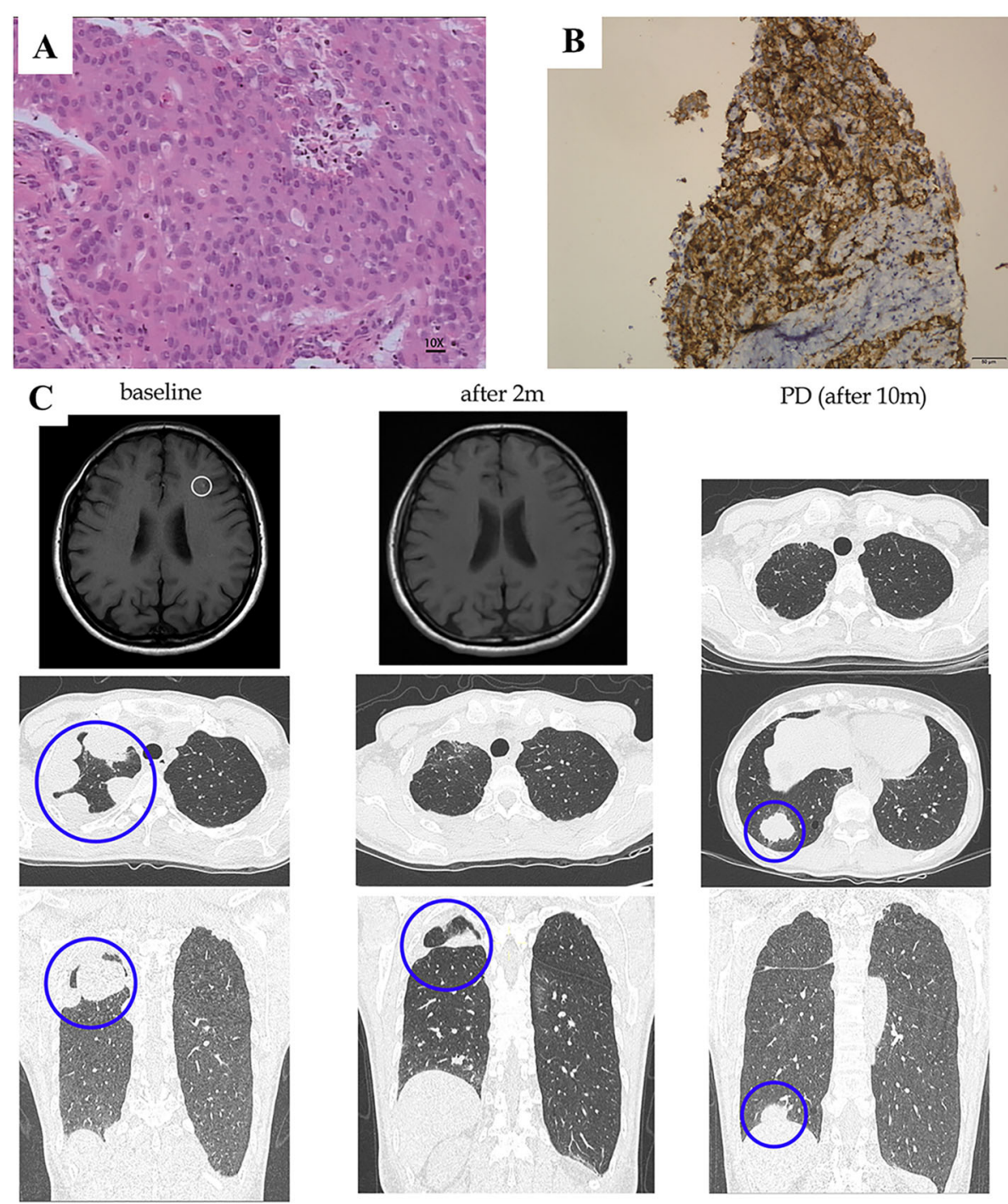

Fig. 5 Response to pembrolizumab in a case with KIF5B-RET fusion and a high level of PD-L1 expression. a Adenocarcinoma nature (10x). b High level of PD-L1 expression (TPS $=50 \%$ ). c Computed tomography scan and magnetic resonance imaging revealing the clinical response to pembrolizumab. Abbreviations: PD - progression disease

of their functions [24], TCR repertoire analysis can be utilized to stratify patients with long survival or screen ICI candidates in the future.

Importantly, our DCR of ICIs is superior to that presented before (60\% vs. $25 \%)$, while PD-L1 expression is similar [17, 42]. Although patients with selected druggable tumor alterations were considered as poor candidates for ICIs (for example, EGFR-mutant and ALKrearranged lung cancers), and diverse efficacy of ICIs in $R E T$-positive patients was reported in previous studies [16], a subgroup of patients exists who can benefit from ICIs as shown in our study. The challenge is how to precisely select these patients in future exploration. In this study, two patients with high PD-L1 expression experienced a satisfying response to ICIs. In a previous study,

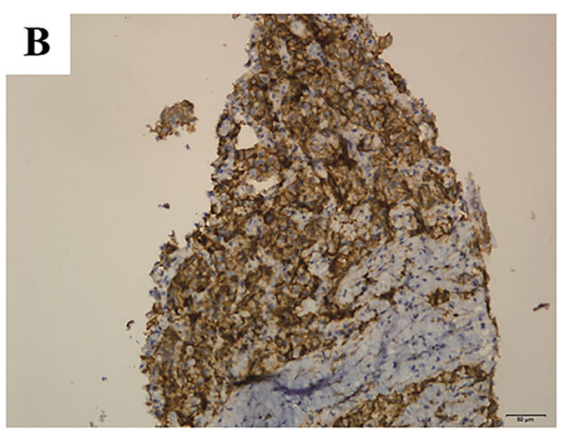

PD (after 10m)

CCDC6-RET was found to be immunogenic because of its peptide level [38]. Predictive immuno-biomarkers are critical. Studies on overall immunogenicity and immune landscape are indispensable to strengthen the full understanding of ICIs in cancers with driver gene alterations.

However, the DCR of ICIs in our study seems to be driven by patients whose best objective response to this treatment was stable disease $(4 / 6)$, and the median PFS is relatively short, revealing suboptimal outcomes of immune checkpoint inhibition. Notably, only one case received pemetrexed plus pembrolizumab as first-line therapy, a combination approved by the FDA based on data from the phase III KEYNOTE-189 trial, but yielded no response. Thus, considering evidence from several studies focusing on ICIs in oncogene-addicted NSCLCs, 
recently a summary of a multidisciplinary roundtable discussion recommended that ICIs should currently only be considered after exhaustion of targeted therapies and chemotherapies in these patients [43].

Our observations can generate meaningful implications for clinical trial settings. Currently, all clinical trials of first-line ICIs, either single- or dual-agent, have excluded EGFR-mutant and $A L K$-rearranged lung cancers but included patients with $R E T$-rearranged lung cancers. In our study, a trend towards inferior outcomes was observed in ICIs compared with chemotherapy. Thus, patients with RET rearrangement might not always be appropriate for first-line immunotherapy trials; they should consider the use of selective targeted therapies (if possible, since more specific and potent TKIs targeting $R E T$ are unavailable for most of our patients at the moment, and the efficacy of MKIs is disappointing) and chemotherapy instead until more specific biomarkers are found to distinguish responders and nonresponders to immunotherapy.

Our study has several limitations. First, dynamic changes in the TCR repertoire are lacking. Next, potential intratumor heterogeneity, evolution during the disease course, and treatment were not addressed by multiregional NGS. Future analysis of NGS data from larger databases is warranted. Moreover, our findings were limited to the relatively small sample size of patients with available treatment data and overlapping in each treatment group.

\section{Conclusions}

In summary, in addition to confirmation of $R E T$-positive lung cancer heterogeneous genotypes and immunotypes, we first reported that patients with concurrent TP53 mutations or high TCR repertoire diversity have relatively unfavorable outcomes. Outcomes with traditional systemic therapies in general are suboptimal. More work is required to understand the biology of $R E T$-rearranged lung cancers and to tailor therapeutic strategies.

\section{Supplementary information}

Supplementary information accompanies this paper at https://doi.org/10. 1186/s13045-020-00866-6.

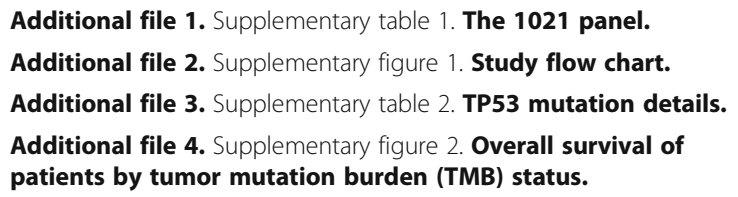

\section{Abbreviations}

CDR: Complementarity-determining regions; DCR: Disease control rate; ICI: Immune checkpoint inhibitor; MKI: Multikinase inhibitors; NGS: Nextgeneration sequencing; NSCLC: Non-small cell lung cancers; ORR: Objective response rate; PBL: Peripheral blood lymphocyte; PFS: Progression-free survival; RECIST: Response Evaluation Criteria in Solid Tumors;
RET: Rearranged during transfection; TCR: T cell receptor; TMB: Tumor mutation burden

\section{Acknowledgements}

We owe thanks to the patients in our study and their family members. We acknowledge the staffs of all centers for their assistance in this study.

\section{Authors' contributions \\ Yi-Long Wu and Jin-Ji Yang designed the investigation and contributed to writing the paper. Xu-Chao Zhang, Hua-Jun Chen, Qing Zhou, Hai-Yan Tu de- signed the investigation and contributed to writing the paper. Xing-Hao Ai, Xiao-Feng Chen, Gai-Li An, Jun Bai, Jin-Lu Shan, Yi-Na Wang, Shuan-Ying Yang, Xiang Liu, Wu Zhuang Hui-Ta Wu, and Bo Zhu performed the investigation. Xue-Feng Xia, Rong-Rong Chen, De-Jian Gu, and Hua-Min Xu provided essential assistance and analyzed data. Jin-Ji Yang, Chang Lu, Xiao-Rong Dong, and Zhao Jun designed and performed the research, supervised the study, analyzed data, and wrote the paper. The authors read and approved the final manuscript.}

\section{Funding}

This work was supported by the National Key Technology R\&D Program of the Ministry of Science and Technology of China: Prevention and Control of Major Non-communicable Diseases [Grant No. 2016YFC 1303304/1303300], the Project of the National Natural Science Funding of China [Grant No. 81472207], National Key R\&D Program of China [Grant No.2016YFC1303800], the Special Fund of Public Interest by the National Health and Family Control Committee [Grant No. 201402031], and the Guangdong Provincial Key Laboratory of Lung Cancer Translational Medicine [Grant No. 2012A061400006/ 2017B030314120].

\section{Availability of data and materials}

The datasets used and analyzed during the current study are available from the corresponding authors on reasonable request.

Ethics approval and consent to participate

Written consent and institutional approval were obtained.

\section{Consent for publication}

All authors read and approved the manuscript as submitted.

\section{Competing interests}

The authors declare that they have no competing interests.

\section{Author details}

${ }^{1}$ The Second School of Clinical Medicine, Southern Medical University, Guangzhou, China. ${ }^{2}$ Guangdong Lung Cancer Institute, Guangdong Provincial People's Hospital, Guangdong Academy of Medical Sciences, Guangzhou, China. ${ }^{3}$ Cancer Center, Union Hospital, Tongji Medical College, Huazhong University of Science and Technology, Wuhan, China. ${ }^{4}$ Key Laboratory of Carcinogenesis and Translational Research (Ministry of Education/Beijing), Department I of Thoracic Oncology, Peking University Cancer Hospital \& Institute, Beijing, China. ${ }^{5}$ Shanghai Lung Cancer Center, Shanghai Chest Hospital, Shanghai Jiao Tong University, Shanghai, China. ${ }^{6}$ Oncology Department, The First Affiliated Hospital of Nanjing Medical University, Nanjing, China. ${ }^{7}$ Department of Clinical Oncology, Shaanxi Provincial People's Hospital, Xi'an, China. ${ }^{8}$ Daping Hospital, Army medical center of PLA, Chongqing, China. 'Department of Oncology, The First Affiliated Hospital of Zhejiang University, Hangzhou, China. ${ }^{10}$ The Second Affiliated Hospital of Xi'an Jiaotong University, Xi'an, China. ${ }^{11}$ Department of Cardiothoracic Surgery, The Second Affiliated Hospital, University of South China, Hengyang, China. ${ }^{12}$ Fujian Provincial Cancer Hospital, Fuzhou, China.

${ }^{13}$ Department of Oncology, Zhongshan Hospital, Xiamen University, Xiamen, China. ${ }^{14}$ Institute of Cancer, Xinqiao Hospital, Third Military Medical

University, Chongqing, China. ${ }^{15}$ Geneplus-Beijing Institute, Beijing, China.

Received: 6 January 2020 Accepted: 27 March 2020

Published online: 15 April 2020

References

1. Takeuchi K, Soda M, Togashi Y, Suzuki R, Sakata S, Hatano S, Asaka R, Hamanaka W, Ninomiya H, Uehara H. RET, ROS1 and ALK fusions in lung cancer. Nat Med. 2012;18:378-81. 
2. Gainor JF, Shaw AT. Novel targets in non-small cell lung cancer: ROS1 and RET Fusions. Oncologist. 2013;18:865-75.

3. Zhang K, Chen H, Wang Y, Yang L, Zhou C, Yin W, Wang G, Mao X, Xiang J, Li B, et al. Clinical characteristics and molecular patterns of RET-rearranged lung cancer in Chinese patients. Oncol Res. 2018. https://doi.org/10.3727/ $096504018 \times 15344979253618$.

4. Michels S, Scheel AH, Scheffler M, Schultheis AM, Gautschi O, Aebersold F, Diebold J, Pall G, Rothschild S, Bubendorf L. Clinicopathological characteristics of RET rearranged lung cancer in European patients. J Thorac Oncol. 2016:11:122-7.

5. Song Z, Yu X, Zhang Y. Clinicopathologic characteristics, genetic variability and therapeutic options of RET rearrangements patients in lung adenocarcinoma. Lung Cancer. 2016;101:16.

6. Tsuta K, Kohno T, Yoshida A, Shimada Y, Asamura H, Furuta K, Kushima R. RET-rearranged non-small-cell lung carcinoma: a clinicopathological and molecular analysis. Br J Cancer. 2014;110:1571-8.

7. Subbiah V, Gainor JF, Rahal R, Brubaker JD, Kim JL, Maynard M, Hu W, Cao Q, Sheets MP, Wilson D. Precision targeted therapy with BLU-667 for RETdriven cancers. Cancer Discov. 2018;8(7):836-49.

8. Subbiah V, Velcheti V, Tuch B, Ebata K, Busaidy N, Cabanillas M, Wirth L, Stock S, Smith S, Lauriault V, et al. Selective RET kinase inhibition for patients with RET-altered cancers. Ann Oncol. 2018;29:1869-76.

9. Zhang C, Leighl NB, Wu Y-L, Zhong W-Z. Emerging therapies for non-small cell lung cancer. J Hematol Oncol. 2019;12:45.

10. Gautschi O, Milia J, Filleron T, Wolf J, Carbone DP, Owen D, Camidge R, Narayanan V, Doebele RC, Besse B. Targeting RET in patients with RETrearranged lung cancers: results from the global, multicenter RET registry. J Clin Oncol. 2017:35:1403-10.

11. Yoh $K$, Seto $T$, Satouchi M, Nishio M, Yamamoto N, Murakami $H$, Nogami N, Matsumoto S, Kohno T, Tsuta K. Vandetanib in patients with previously treated RET-rearranged advanced non-small-cell lung cancer (LURET): an open-label, multicentre phase 2 trial. Lancet Respir Med. 2017;5:42-50

12. Lee SH, Lee JK, Ahn MJ, Kim DW, Sun JM, Keam B, Kim TM, Heo DS, Ahn JS, Choi YL. Vandetanib in pretreated patients with advanced non-small cell lung cancer harboring RET rearrangement: a phase II clinical trial. Ann Oncol. 2016;28:292.

13. Drilon A, Rekhtman N, Arcila M, Wang L, Ni A, Albano M, Van VM, Somwar R, Smith RS, Montecalvo J. Cabozantinib in patients with advanced RETrearranged non-small-cell lung cancer: an open-label, single-centre, phase 2, single-arm trial. Lancet Oncol. 2016;17:1653.

14. Drilon A, Lin J, Filleron T, Ni A, Milia J, Bergagnini I, Hatzoglou V, Velcheti V, Offin $M, L i B$, et al. Frequency of brain metastases and multikinase inhibitor outcomes in patients with RET-rearranged lung cancers. A Drilon A. J Thorac Oncol. 2018:13:1595-601.

15. Drilon A, Bergagnini I, Delasos L, Sabari J, Woo K, Plodkowski A, Wang L, Hellmann M, Joubert P, Sima C, et al. Clinical outcomes with pemetrexedbased systemic therapies in RET-rearranged lung cancers. Ann Oncol. 2016; 27:1286-91.

16. Sarfaty M, Moore A, Neiman V, Dudnik E, llouze M, Gottfried M, Katznelson R, Nechushtan H, Sorotsky HG, Paz K. RET fusion lung carcinoma: response to therapy and clinical features in a case series of 14 patients. Clin Lung Cancer. 2017:18:e223.

17. Mazieres J, Drilon A, Lusque A, Mhanna L, Cortot AB, Mezquita L, Thai AA, Mascaux C, Couraud S, Veillon R, et al. Immune checkpoint inhibitors for patients with advanced lung cancer and oncogenic driver alterations: results from the IMMUNOTARGET registry. Ann Oncol. 2019; 30:1321-8.

18. Su S, Dong Z-Y, Xie Z, Yan L-X, Li Y-F, Su J, Liu S-Y, Yin K, Chen R-L, Huang $S-M$, et al. Strong PD-L1 expression predicts poor response and de novo resistance to EGFR TKIs among non-small cell lung cancer patients with EGFR mutation. J Thorac Oncol. 2018;13(11):1668-75.

19. Li H, Durbin R. Fast and accurate short read alignment with BurrowsWheeler transform. Bioinformatics. 2009:25:1754-60.

20. Cibulskis K, Lawrence MS, Carter SL, Sivachenko A, Jaffe D, Sougnez C, Gabriel S, Meyerson M, Lander ES, Getz G. Sensitive detection of somatic point mutations in impure and heterogeneous cancer samples. Nat Biotechnol. 2013:31:213-9.

21. Li J, Lupat R, Amarasinghe KC, Thompson ER, Doyle MA, Ryland GL, Tothill RW, Halgamuge SK, Campbell IG, Gorringe KL. CONTRA: copy number analysis for targeted resequencing. Bioinformatics. 2012;28:1307-13.
22. Zhang Y, Chang L, Yang Y, Fang W, Guan Y, Wu A, Hong S, Zhou H, Chen $\mathrm{G}$, Chen $\mathrm{X}$, et al. The correlations of tumor mutational burden among single-region tissue, multi-region tissues and blood in non-small cell lung cancer. J Immunother Cancer. 2019;7:98.

23. Gibney GT, Weiner LM, Atkins MB. Predictive biomarkers for checkpoint inhibitor-based immunotherapy. Lancet Oncol. 2016;17:e542-51.

24. Hofman P, Heeke S, Alix-Panabières C, Pantel K. Liquid biopsy in the era of immuno-oncology: is it ready for prime-time use for cancer patients? Ann Oncol. 2019;30:1448-59.

25. Tumeh PC, Harview CL, Yearley JH, Shintaku IP, Taylor EJ, Robert L, Chmielowski B, Spasic M, Henry G, Ciobanu V. PD-1 blockade induces responses by inhibiting adaptive immune resistance. Nature. 2014;515:568.

26. Postow MA, Manuel M, Wong P, Yuan J, Dong Z, Liu C, Perez S, Tanneau I Noel M, Courtier A. Peripheral T cell receptor diversity is associated with clinical outcomes following ipilimumab treatment in metastatic melanoma. J Immunother Cancer. 2015;3:23.

27. Liu YY, Yang QF, Yang JS, Cao RB, Liang JY, Liu YT, Zeng YL, Chen S, Xia XF, Zhang K. Characteristics and prognostic significance of profiling the peripheral blood T-cell receptor repertoire in patients with advanced lung cancer. Int J Cancer. 2019;145(5):1423-31.

28. Hogan SA, Courtier A, Cheng PF, Jaberg-Bentele NF, Goldinger SM, Manue M, Perez S, Plantier N, Mouret J-F, Nguyen-Kim TDL. Peripheral blood TCR repertoire profiling may facilitate patient stratification for immunotherapy against melanoma. Cancer Immunol Res. 2019;7:77-85.

29. Rosati E, Dowds CM, Liaskou E, Henriksen EKK, Karlsen TH, Franke A. Overview of methodologies for T-cell receptor repertoire analysis. BMC Biotechnol. 2017;17:61.

30. Liu YY, Yang QF, Yang JS, Cao RB, Liang JY, Liu YT, Zeng YL, Chen S, Xia XF, Zhang K, Liu L. Characteristics and prognostic significance of profiling the peripheral blood T-cell receptor repertoire in patients with advanced lung cancer. Int J Cancer. 2019;145:1423-31.

31. Kirsch I, Vignali M, Robins H. T-cell receptor profiling in cancer. Mol Oncol. 2015;9:2063-70.

32. Reuben A, Gittelman R, Gao J, Zhang J, Yusko EC, Wu C-J, Emerson R, Zhang J, Tipton C, Li J, et al. TCR repertoire intratumor heterogeneity in localized lung adenocarcinomas: an association with predicted neoantigen heterogeneity and postsurgical recurrence. Cancer Discov. 2017;7:1088-97.

33. Canale M, Petracci E, Delmonte A, Chiadini E, Dazzi C, Papi M, Capelli L, Casanova C, De Luigi N, Mariotti M, et al. Impact of TP53 Mutations on Outcome in EGFR-Mutated Patients Treated with First-Line Tyrosine Kinase Inhibitors. Clin Cancer Res. 2017;23:2195-202.

34. VanderLaan PA, Rangachari D, Mockus SM, Spotlow V, Reddi HV, Malcolm J, Huberman MS, Joseph $\sqcup$, Kobayashi SS, Costa DB. Mutations in TP53, PIK3CA, PTEN and other genes in EGFR mutated lung cancers: correlation with clinical outcomes. Lung Cancer. 2017;106:17-21.

35. Christopoulos P, Kirchner M, Bozorgmehr F, Endris V, Budczies J, Ristau J, Penzel R, Herth F, Heussel CP, Eichhorn M, et al. Identification of a highly lethal V3 + TP53 + subset in ALK + lung adenocarcinoma. Int J Cancer. 2018;144:190-99.

36. Kron A, Alidousty C, Scheffler M, Merkelbach-Bruse S, Seidel D, Riedel R, Ihle MA, Michels S, Nogova L, Fassunke J, et al. Impact of TP53 mutation status on systemic treatment outcome in ALK-rearranged non-small-cell lung cancer. Ann Oncol. 2018:29:2068-75.

37. Brosh R, Rotter $\mathrm{V}$. When mutants gain new powers: news from the mutant p53 field. Nat sssRev Cancer. 2009:9:701-13.

38. Gao Q, Liang WW, Foltz SM, Mutharasu G, Jayasinghe RG, Cao S, Liao WW, Reynolds SM, Wyczalkowski MA, Yao L. Driver fusions and their implications in the development and treatment of human cancers. Cell Rep. 2018:23:227.

39. Wang C, Yin R, Dai J, Gu Y, Cui S, Ma H, Zhang Z, Huang J, Qin N, Jiang T, et al. Whole-genome sequencing reveals genomic signatures associated with the inflammatory microenvironments in Chinese NSCLC patients. Nat Commun. 2018;9:2054

40. Simoni $Y$, Becht E, Fehlings M, Loh CY, Koo S-L, Teng KWW, Yeong JPS, Nahar R, Zhang T, Kared H. Bystander CD8+ T cells are abundant and phenotypically distinct in human tumour infiltrates. Nature. 2018;557: 575.

41. Scheper W, Kelderman S, Fanchi LF, Linnemann C, Bendle G, de Rooij MA, Hirt C, Mezzadra R, Slagter M, Dijkstra K. Low and variable tumor reactivity of the intratumoral TCR repertoire in human cancers. Nat Med. 2019;25:89. 
42. Offin M, Guo R, Wu SL, Sabari J, Land JD, Ni A, Montecalvo J, Halpenny DF, Buie LW, Pak T, et al. Immunophenotype and Response to Immunotherapy of RET -Rearranged Lung Cancers. JcO Precis Oncol. 2019;3:1-8.

43. Berghoff AS, Bellosillo B, Caux C, de Langen A, Mazieres J, Normanno N, Preusser M, Provencio M, Rojo F, Wolf J. Immune checkpoint inhibitor treatment in patients with oncogene-addicted non-small cell lung cancer (NSCLC): summary of a multidisciplinary round-table discussion. ESMO Open. 2019;4:e000498.

\section{Publisher's Note}

Springer Nature remains neutral with regard to jurisdictional claims in published maps and institutional affiliations.

Ready to submit your research? Choose BMC and benefit from:

- fast, convenient online submission

- thorough peer review by experienced researchers in your field

- rapid publication on acceptance

- support for research data, including large and complex data types

- gold Open Access which fosters wider collaboration and increased citations

- maximum visibility for your research: over $100 \mathrm{M}$ website views per year

At $\mathrm{BMC}$, research is always in progress.

Learn more biomedcentral.com/submissions 